\title{
Density functional theory and multiscale materials modeling*
}

\author{
SWAPAN K GHOSH \\ Chemistry Group, Bhabha Atomic Research Centre, Mumbai 400 085, India
}

\begin{abstract}
One of the vital ingredients in the theoretical tools useful in materials modeling at all the length scales of interest is the concept of density. In the microscopic length scale, it is the electron density that has played a major role in providing a deeper understanding of chemical binding in atoms, molecules and solids. In the intermediate mesoscopic length scale, an appropriate picture of the equilibrium and dynamical processes has been obtained through the single particle number density of the constituent atoms or molecules. A wide class of problems involving nanomaterials, interfacial science and soft condensed matter has been addressed using the density based theoretical formalism as well as atomistic simulation in this regime. In the macroscopic length scale, however, matter is usually treated as a continuous medium and a description using local mass density, energy density and other related density functions has been found to be quite appropriate. A unique single unified theoretical framework that emerges through the density concept at these diverse length scales and is applicable to both quantum and classical systems is the so called density functional theory (DFT) which essentially provides a vehicle to project the many-particle picture to a single particle one. Thus, the central equation for quantum DFT is a one-particle Schrödinger-like Kohn-Sham equation, while the same for classical DFT consists of Boltzmann type distributions, both corresponding to a system of noninteracting particles in the field of a density-dependent effective potential. Selected illustrative applications of quantum DFT to microscopic modeling of intermolecular interaction and that of classical DFT to a mesoscopic modeling of soft condensed matter systems are presented.
\end{abstract}

Keywords. Density functional theory; soft condensed matter; materials modeling.

\section{Introduction}

Materials modeling and simulation form important areas of research aiming at designing materials with tailored properties for desired applications. Although at the fundamental level, the properties of materials are dictated by the basic laws of quantum mechanics, their prediction through a direct $a b$ initio electronic structure calculation is often too ambitious even with the state of the art computational resources. One can, however, view the materials through the windows of different length scales and develop modeling in three broad domains, viz. microscopic, mesoscopic and macroscopic regimes covering the length scales of $0 \cdot 1-1 \mathrm{~nm}, 1-100 \mathrm{~nm}$ and larger than $100 \mathrm{~nm}$, respectively.

In the macroscopic length scales, matter is considered as a continuous medium and the theoretical tools used are the conventional approaches of continuum mechanics and hydrodynamics of classical physics. The property parameters needed in this approach as input can be obtained from a consideration of the so called mesoscopic length scale with an atomistic description using classical and statistical mechanics of interacting particles (atoms and molecules), their movements and distributions. The major

*Dedicated to Prof. B M Deb on the occasion of his 60th birthday. input parameters in this intermediate length scale are the interparticle interactions which can be generated through a quantum mechanical investigation within the microscopic length scale. In this shorter length scale, one can deal with the interacting electrons and the nuclei, employing a complete $a b$ initio electronic structure description within the framework of available approximate theories.

Although the electronic, atomistic and continuum descriptions for materials modeling at the three respective length scales appear to be quite diverse, there lies a unified theoretical framework which can encompass all of them and the present work aims at presenting a glimpse of this area of research. The concept that plays the role of a common underlying thread in this endeavour is the concept of density and the associated theoretical approach is the so called density functional theory (DFT) (Hohenberg and Kohn 1964; Kohn and Sham 1965; Mermin 1965; Parr and Yang 1989, 1995).

In the microscopic length scale, the DFT based description of a many-electron system employs the single-particle electron density (Ghosh and Deb 1982; March and Deb 1987) as the basic variable bypassing the manyelectron wavefunction, providing thereby tremendous simplification, computational economy and foundation of widely used chemical concepts. DFT has also been formulated (Henderson 1992) for the mesoscopic length 
scale, with the density distribution of the atomistic particles as the basic variable, leading to wide applications in the field of soft condensed matter (deGennes 1992, 1999) physics. The recent upsurge in nanomaterials research has also led to greater opportunities for materials modeling in this mesoscopic size regime. The effective interparticle interaction potentials needed for the mesoscopic DFT can be obtained through the microscopic or quantum DFT either through a detailed electron density calculation or a modeling of chemical binding using DFT based atomic parameters. One can also couple the interatomic potential calculation based on quantum DFT for electrons with the classical mesoscopic DFT for the atomistic particles in the spirit of the well known Car and Parrinello (1985) approach for DFT based $a b$ initio simulation. For simplicity, one can also employ empirical pair potentials in the mesoscopic DFT as is often done in usual classical simulation. In the domain of macroscopic length scale, a density based continuous medium description is possible using the mass, current, energy and other property densities as the basic variables, reminiscent of the classical hydrodynamics.

Thus, in DFT as applied to materials modeling in the short, intermediate and large length scales, the respective basic variables are the electron density, the single particle density and the property density, corresponding to the electron, atom and the volume element as the respective building blocks. A central common feature in DFT is the collection of building blocks with an inhomogeneous density distribution arising due to the field of an external potential. However, there are many phenomena such as freezing, nucleation, crystallization etc where the density inhomogeneity arises through stabilization of thermal density fluctuations even in absence of external potentials.

Besides the DFT corresponding to different individual length scales, many of the areas of materials science and engineering dealing with the material properties needs a bridging of the microscopic, mesoscopic and macroscopic length scales. Building such bridges can enhance the possibility of opening new windows to view the details of the microstructure which determines the properties of different forms of matter that matters in many applications of interest.

In what follows, we aim at presenting a unified view of DFT encompassing all these diverse situations and length scales dealing with quantum as well as classical systems and electronic, atomistic and macroscopic density concepts (Ghosh 2002). Thus, in $\$ 2$, we first discuss the basic theoretical formalism of quantum and classical DFT as used in different regimes of length scales and then consider in $\$ 3$, a specific application of quantum DFT for the modeling of chemical binding to obtain the interatomic potential that can be used in the mesoscopic DFT. The DFT based modeling in classical complex fluids or soft matter is presented in $\S 4$. Extension beyond the equilibrium situations covering some of the dynamical aspects is discussed in $\$ 5$ and finally we offer a few concluding remarks in $\S 6$.

\section{Density functional theory of quantum and classical systems: a unified view}

The single-particle density $\rho(\mathbf{r})$, which is the basic variable in DFT, is defined, for an $N$-particle quantum or classical system, by integrating the full distribution function, $P\left(\mathbf{r}_{1}, \mathbf{r}_{2}, \ldots, \mathbf{r}_{N}\right)$ over $N-1$ variables, as

$$
\rho\left(\mathbf{r}_{1}\right)=N \int \cdots \int \mathrm{d} \mathbf{r}_{2} \mathrm{~d} \mathbf{r}_{3} \cdots \mathrm{d} \mathbf{r}_{N} P\left(\mathbf{r}_{1}, \mathbf{r}_{2}, \ldots, \mathbf{r}_{N}\right) .
$$

For a quantum system, the distribution function, $P\left(\mathbf{r}_{1}\right.$, $\left.\mathbf{r}_{2}, \ldots, \mathbf{r}_{N}\right)$ is given by $\left|\psi\left(\mathbf{r}_{1}, \mathbf{r}_{2}, \ldots, \mathbf{r}_{N}\right)\right|^{2}$, where $\boldsymbol{\psi}$ is the many-electron wavefunction. The density variable which is a function in three-dimensional space and represents locally the number density clearly satisfies the normalization $\int \rho(\mathbf{r}) \mathrm{d} \mathbf{r}=N$. For interacting particles, the possibility of describing a quantum or classical many-particle system completely in terms of the single-particle density is not obvious and has been established rigorously through the theorems proved by Hohenberg and Kohn (1964) and Mermin (1965) (HKM).

Although the birth of a formal DFT is rather recent, approximate versions of DFT have existed for a very long time in the forms of the Thomas Fermi (TF) (Thomas 1927; Fermi 1928) and Debye-Huckel (DH) (1923) theories for quantum and classical systems, respectively dealing with the distribution of electrons around the nuclei in atoms and that of ions in electrolyte solutions. Inspite of a wide difference in the nature of these systems, the two approximate theoretical descriptions have close similarities. Thus, in both cases, the electrostatic potential, $\phi \mathbf{r})$, is defined through the Poisson equation

$$
\nabla^{2} \phi(\mathbf{r})=-4 \pi e^{2} \rho(\mathbf{r}) .
$$

The TF theory corresponds to the additional relation between the potential and the electron density as given by the TF equation, viz.

$$
\frac{5}{3} \frac{\hbar^{2}}{m} C_{\mathrm{F}} \boldsymbol{\rho}(\mathbf{r})^{2 / 3}+\phi(\mathbf{r})=\mu=0 ; C_{\mathrm{F}}=\frac{3}{10}\left(3 \pi^{2}\right)^{2 / 3},
$$

corresponding to the Fermi-Dirac statistics of quantum systems, while in DH theory, this relation is the classical Boltzmann distribution for the ions given by

$$
\rho_{\alpha}(\mathbf{r})=\rho_{\alpha}^{0} \exp \left[-\beta_{0} q_{\alpha} \phi(\mathbf{r})\right],
$$

where $\beta_{0}\left(=1 / k_{\mathrm{B}} T\right)$ represents the inverse temperature with $k_{\mathrm{B}}$ as the Boltzmann constant. The charge density in the second case is defined from the component densities $\rho_{\alpha}(\mathbf{r})$ as 


$$
\rho(\mathbf{r})=\sum_{\alpha} q_{\alpha} \rho_{\alpha}(\mathbf{r})
$$

with $q_{\alpha}$ denoting the ionic charge of the $\alpha$ th component. Both these theories can, however, be obtained as well defined approximations of the exact HKM versions of DFT.

For a many-particle system characterized by an external potential $v(\mathbf{r})$ (arising due to the nuclei in the case of electrons and due to the walls or pores in the case of confined classical fluid particles), the proofs of HKM follow by establishing a one to one mapping between the density and the potential. In the resulting formal DFT, the ground state energy, $E_{v}[\rho]$ (for a many-electron quantum system) or, the grand potential $\Omega_{\nu}[\rho]$ (for a many-particle classical system) is treated as the unique functionals of density, given respectively by

$$
E_{v}[\boldsymbol{\rho}]=F[\boldsymbol{\rho}]+\int \mathrm{d} \mathbf{r} \boldsymbol{\rho}(\mathbf{r}) v(\mathbf{r}),
$$

and

$$
\Omega_{v}[\rho]=F[\rho]+\int \operatorname{dr} \rho(\mathbf{r})[v(\mathbf{r})-\mu],
$$

with $\mu$ denoting the chemical potential. The true equilibrium densities in both cases are determined by the Euler-Lagrange equation corresponding to the minima of the respective energy density functionals, as given by

$$
\mu=v(\mathbf{r})+\frac{\delta F[\rho]}{\delta \rho(\mathbf{r})}
$$

For many-electron systems, the functional $F[\rho]$ is universal and can be expressed as

$$
F[\rho]=T_{\mathrm{s}}[\rho]+E_{\text {coul }}[\rho]+E_{\mathrm{xc}}[\rho],
$$

where $T_{\mathrm{s}}[\rho], \quad E_{\mathrm{coul}}[\rho]$ and $E_{\mathrm{xc}}[\rho]$ represent the noninteracting kinetic energy, classical Coulomb energy and the exchange-correlation (XC) energy density functionals, respectively.

For a classical fluid with inhomogeneous density distribution, the functional $F[\rho]$ representing the intrinsic Helmholtz free energy is universal only for a specified interparticle interaction and can be expressed as

$$
F[\rho]=F_{\text {id }}[\rho]+F_{\text {ex }}[\rho],
$$

where $F_{\mathrm{id}}[\rho]$ represents the ideal-gas free-energy functional corresponding to absence of internal interactions and is the analogue of the noninteracting kinetic energy functional, $T_{\mathrm{s}}[\rho]$ of (8) for quantum systems, while $F_{\text {ex }}[\rho]$ representing the excess free-energy for the classical system is analogous to the interaction energy functional $\left(E_{\text {coul }}[\rho]+E_{\mathrm{xc}}[\rho]\right)$ of a quantum system.

For quantum systems, a minimization of the energy functional $E_{\nu}[\rho]$ with the known exact expression of $E_{\text {coul }}[\rho]$ given by

$$
E_{\text {coul }}[\rho]=\frac{e^{2}}{2} \iint \mathrm{d} \mathbf{r} \mathrm{d} \mathbf{r}^{\prime} \frac{\rho(\mathbf{r}) \rho\left(\mathbf{r}^{\prime}\right)}{\left|\mathbf{r}-\mathbf{r}^{\prime}\right|},
$$

with neglect of $E_{\mathrm{xc}}[\rho]$ and the so called local density approximation for the kinetic energy functional as $T_{\mathrm{s}}[\rho]=$ $C_{\mathrm{F}}\left(\hbar^{2} / m\right) \int \mathrm{d} \mathbf{r} \rho(\mathbf{r})^{5 / 3}$, leads to the TF theory for the electron density given by (3). On the other hand, using the known exact expression for $T_{\mathrm{s}}[\rho]$ as given by

$$
T_{\mathrm{s}}[\boldsymbol{\rho}]=-\frac{\hbar^{2}}{2 m} \sum_{i}\left\langle\psi_{i}(\mathbf{r})\left|\nabla^{2}\right| \psi_{i}(\mathbf{r})\right\rangle
$$

in the energy functional $E_{\nu}[\rho]$, one obtains, on minimization, the effective one-particle Kohn-Sham (1965) equation, viz.

$$
\left[-\frac{\hbar^{2}}{2 m} \nabla^{2}+v_{\mathrm{eff}}(\mathbf{r} ;[\boldsymbol{\rho}])\right] \boldsymbol{\psi}_{i}=\boldsymbol{\varepsilon}_{i} \boldsymbol{\psi}_{i}
$$

where the orbitals $\left\{\psi_{i}(\mathbf{r})\right\}$ define the density $\rho(\mathbf{r})$ as the sum $\rho(\mathbf{r})=\sum_{i}\left|\Psi_{i}(\mathbf{r})\right|^{2}$ and the effective potential is given by the density functional as

$$
v_{\text {eff }}(\mathbf{r} ;[\boldsymbol{\rho}])=v(\mathbf{r})+e^{2} \int \mathrm{d} \mathbf{r}^{\prime} \frac{\rho\left(\mathbf{r}^{\prime}\right)}{\left|\mathbf{r}-\mathbf{r}^{\prime}\right|}+\frac{\delta E_{\mathrm{xc}}[\mathbf{\rho}]}{\delta \mathbf{\rho}(\mathbf{r})} .
$$

Analogously, for a classical system, on using the exact expression for the ideal-gas free-energy functional, $F_{\mathrm{id}}[\rho]$, as given by

$$
F_{\text {id }}[\rho]=\frac{1}{\beta_{0}} \int \mathrm{d} \mathbf{r} \rho(\mathbf{r})\left\{\ln \left[\rho(\mathbf{r}) \Lambda^{3}\right]-1\right\},
$$

with $\Lambda$ being the thermal de-Broglie wavelength, the Euler-Lagrange equation (7) leads to the density equation

$$
\rho(\mathbf{r})=\Lambda^{-3} \exp \left(\beta_{0} \mu\right) \exp \left\{-\beta_{0} v(\mathbf{r})+c^{(1)}(\mathbf{r} ;[\rho])\right\},
$$

representing essentially a Boltzmann-like distribution of an ideal gas in the field of an effective potential $v_{\text {eff }}(\mathbf{r} ;[\boldsymbol{\rho}])=\left[v(\mathbf{r})-\beta_{0}^{-1} c^{(1)}(\mathbf{r})\right]$. Here the first order direct correlation function $(\mathrm{DCF}) c^{(1)}(\mathbf{r})$ defined as

$$
c^{(1)}(\mathbf{r})=-\beta_{0} \frac{\delta F_{\mathrm{ex}}[\rho]}{\delta \rho(\mathbf{r})},
$$

is the first member of the $n$th order DCF defined as the functional derivative of the excess free energy, $F_{\mathrm{ex}}[\rho]$, as

$$
c^{(n)}\left(\mathbf{r}_{1}, \mathbf{r}_{2}, \ldots, \mathbf{r}_{N}\right)=-\beta_{0} \frac{\delta^{n} F_{\mathrm{ex}}[\rho]}{\delta \rho\left(\mathbf{r}_{1}\right) \delta \rho\left(\mathbf{r}_{2}\right) \cdots \delta\left(\mathbf{r}_{n}\right)},
$$

and provides the extra contribution to the effective potential, arising from interparticle interactions and correlations in analogy to the exchange-correlation contribution to the Kohn-Sham potential for quantum systems. The $\mathrm{DH}$ theory can be obtained from an extension of this exact classical DFT to a two-component ionic system 
with an approximation of the excess free energy functional as the pure Coulomb contribution analogous to (10).

It is thus clear that DFT provides a formally exact single-particle framework for many-particle systems, providing the density distribution through that of a system of noninteracting particles, viz. the one-particle Schrödinger equation for a quantum system and Boltzmann-like distribution for classical particles. This has emerged through an exact mapping of the actual system of $N$ interacting particles in the field of an external potential, $v(\mathbf{r})$ to another model system of $N$ noninteracting particles of the same density, $\boldsymbol{\rho}(\mathbf{r})$ but moving in an effective potential $v_{\text {eff }}(\mathbf{r} ;[\boldsymbol{\rho}])$, itself depending on the density, thus requiring a self-consistent iterative procedure for solving the resulting equations.

Although the DFT framework is exact, the crux of the problem lies in the fact that the exact form of the density functionals is not known for an inhomogeneous density distribution and hence approximations are needed for the $\mathrm{XC}$ energy functional, $E_{\mathrm{xc}}[\rho]$ for quantum systems, and the excess free energy functional $F_{\mathrm{ex}}[\rho]$ or its derivatives for classical systems.

The approximate procedures usually are based on the knowledge of the functionals for the corresponding systems with homogeneous density. The simplest approximation scheme is the so called local density approximation (LDA) where the expression for the energy functional of the homogeneous system is directly evaluated using the inhomogeneous density. For quantum systems, the XC energy functional is thus approximated as

$$
E_{\mathrm{xc}}^{\mathrm{LDA}}[\rho(\mathbf{r})]=\int \mathrm{d} \mathbf{r} \rho(\mathbf{r}) \tilde{\varepsilon}_{\mathrm{xc}}(\rho(\mathbf{r})),
$$

where $\tilde{\varepsilon}_{\mathrm{xc}}$ denotes the XC energy per electron for the homogeneous electron gas. The LDA, however, cannot be employed for approximating the excess free energy of the classical systems such as liquids where the particle sizes are finite. For these systems, the excess free energy, $F_{\text {ex }}$ or the first order DCF, $c^{(1)}$ are approximated by evaluating the corresponding expressions for $\tilde{f}_{\mathrm{ex}}$ or $\tilde{c}^{(1)}$ of the homogeneous fluid as

$$
\begin{aligned}
& F_{\mathrm{ex}}[\boldsymbol{\rho}(\mathbf{r})]=\int \mathrm{d} \mathbf{r} \rho(\mathbf{r}) \tilde{f}_{\mathrm{ex}}(\overline{\boldsymbol{\rho}}(\mathbf{r})), \\
& c^{(1)}(\mathbf{r} ;[\rho(\mathbf{r})])=\tilde{c}^{(1)}(\bar{\rho}(\mathbf{r})),
\end{aligned}
$$

by using an effective density $\bar{\rho}(\mathbf{r})$ obtained by coarse graining of the actual density distribution, $\rho(\mathbf{r})$, with a suitable weight function, $w\left(\mathbf{r}, \mathbf{r}^{\prime} ; \overline{\boldsymbol{\rho}}(\mathbf{r})\right)$ as

$$
\overline{\boldsymbol{\rho}}(\mathbf{r})=\int \mathrm{d} \mathbf{r}^{\prime} \rho\left(\mathbf{r}^{\prime}\right) w\left(\mathbf{r}, \mathbf{r}^{\prime} ; \overline{\mathbf{\rho}}(\mathbf{r})\right)
$$

This procedure forms the basis of the so called weighted density approximation (WDA) (Tarazona 1984, 1985;
Denton and Ashcroft 1989; Evans 1992) where the slowly varying nature of the effective density takes care of the difficulties arising in applying LDA directly to these systems (particularly regions where the densities might exceed the density of close packing). Other procedures include improvements of LDA by including the gradient corrections for quantum systems (Lee et al 1988; Becke 1993) and functional perturbation techniques (Choudhury and Ghosh 1998a, 1999) in the theory of fluids.

Although the discussion so far has been restricted to a single density quantity, it may be noted that the spinpolarized quantum systems and the classical two-component fluid mixtures can also be treated through analogous unified DFT descriptions by considering the two-spin components of the density and the individual component densities as the respective basic variables. In the following sections, we consider specific applications of these generalized DFT for two-component systems for quantum as well as classical systems.

\section{Quantum DFT and microscopic modeling of interatomic interaction potential}

The electron density based quantum DFT has been highly successful in the electronic structure calculation of atoms, molecules, clusters and solids. The interatomic or intermolecular interaction potential obtained through such an $a b$ initio calculation using suitable XC energy functionals can be used in the study of mesoscopic DFT or computer simulation in the atomistic length scale. In this work, however, we discuss a simpler and appealing alternative model which is based on the conceptual aspects of quantum DFT, the basic foundation of which lies in the chemical potential equalization (Parr et al 1978) within a many-electron system as indicated in (7) which can be generalized to the spin-polarized situation (Ghosh 1994; Ghanty and Ghosh 1994a) as

$$
\begin{aligned}
& \mu_{\alpha}=\frac{\delta E\left[\rho_{\alpha}, \rho_{\beta}\right]}{\delta \rho_{\alpha}(\mathbf{r})}=v(\mathbf{r})+\frac{\delta F\left[\rho_{\alpha}, \rho_{\beta}\right]}{\delta \rho_{\alpha}(\mathbf{r})}, \\
& \mu_{\beta}=\frac{\delta E\left[\rho_{\alpha}, \rho_{\beta}\right]}{\delta \rho_{\beta}(\mathbf{r})}=v(\mathbf{r})+\frac{\delta F\left[\rho_{\alpha}, \rho_{\beta}\right]}{\delta \rho_{\beta}(\mathbf{r})}
\end{aligned}
$$

where $\mu_{\alpha}$ and $\mu_{\beta}$ are the up- and down-spin chemical potentials which correspond to the spin-polarized version of the energy functional of (5) expressed as functionals of the two densities, $\rho_{\alpha}(\mathbf{r})$ and $\rho_{\beta}(\mathbf{r})$, as

$$
E\left[\boldsymbol{\rho}_{\alpha}, \rho_{\beta}\right]=F\left[\rho_{\alpha}, \rho_{\beta}\right]+\int d \mathbf{r}\left[\rho_{\alpha}(\mathbf{r})+\rho_{\beta}(\mathbf{r})\right] v(\mathbf{r}) .
$$

We propose to obtain the energy change in a many-electron system arising from a reorganization of its electron 
density (York and Yang 1996; Wadehra and Ghosh 2002) through a functional Taylor expansion (up to second order) in terms of the density changes, $\oint_{v}(\mathbf{r})$ (for $v=\alpha, \beta)$, as

$$
\begin{aligned}
& \Delta E=\sum_{v} \int \mathrm{d} \mathbf{r}\left(\frac{\delta E\left[\rho_{\alpha}, \rho_{\beta}\right]}{\delta \rho_{v}(\mathbf{r})}\right) \delta \rho_{v}(\mathbf{r}) \\
& +\frac{1}{2} \sum_{\mu} \sum_{v} \iint \mathrm{d} \mathbf{r} d \mathbf{r}^{\prime}\left(\frac{\delta^{2} E\left[\rho_{\alpha}, \rho_{\beta}\right]}{\delta \rho_{\mu}(\mathbf{r}) \delta \rho_{v}\left(\mathbf{r}^{\prime}\right)}\right) \delta \rho_{\mu}(\mathbf{r}) \delta \boldsymbol{\rho}_{(}\left(\mathbf{r}^{\prime}\right)
\end{aligned}
$$

The corresponding changes in the two chemical potentials, $\mu_{\alpha}^{0}$ and $\mu_{\beta}^{0}$, of the unperturbed system can be expressed as

$$
\mu_{\alpha}=\mu_{\alpha}^{0}+\sum_{v} \int d \mathbf{r}^{\prime} \eta_{\alpha v}\left(\mathbf{r}, \mathbf{r}^{\prime}\right) \delta p_{\nu}\left(\mathbf{r}^{\prime}\right)
$$

with a similar expression for $\mu_{\beta}$ obtained by replacing $\alpha$ with $\beta$ Here the hardness kernel (Berkowitz et al 1985; Ghosh 1990), $\eta_{\mu v}\left(\mathbf{r}, \mathbf{r}^{\prime}\right)$, represents the energy functional derivative

$$
\eta_{\mu v}\left(\mathbf{r}, \mathbf{r}^{\prime}\right)=\left(\frac{\delta^{2} F\left[\rho_{\alpha}, \rho_{\beta}\right]}{\delta \rho_{\mu}(\mathbf{r}) \delta \rho_{v}\left(\mathbf{r}^{\prime}\right)}\right)
$$

We now specialize to the case of a set of atoms (say, $M$ in number) located at the fixed positions $\left\{\mathbf{R}_{i}\right\}$ corresponding to a particular (say, equilibrium) configuration and express the density change, $\oint_{v}(\mathbf{r})$ for $v=\alpha, \beta$ due to the interaction between the atoms by partitioning as a sum of the atomic contributions as

$$
\delta \boldsymbol{q}(\mathbf{r})=\sum_{i} \delta \boldsymbol{\rho}_{v, i}(\mathbf{r})=\sum_{i} \delta \boldsymbol{q}_{, i}\left(\mathbf{r}_{i}\right)
$$

with $\mathbf{r}_{i}$ denoting the atomic region around the $i$ th atomic site location, $\mathbf{R}_{i}$. Without loss of generality, for simplicity, we assume the density components, $\oint_{v, i}(\mathbf{r})$, to vanish outside this region, $\mathbf{r}_{i}$ belonging to the $i$ th site. The objective is to develop a lattice model of the interacting atoms under consideration with the lattice sites given by their locations for which the expression of the chemical potential given by (26) recast to obtain the same for the $i$ th atomic site is given by

$$
\mu_{\alpha}(i)=\mu_{\alpha}^{0}(i)+\sum_{v} \sum_{j} \int \mathrm{d} \mathbf{r}_{j}^{\prime} \eta_{\mathbf{\alpha} v}\left(\mathbf{R}_{i}, \mathbf{r}_{j}^{\prime}\right) \delta \mathbf{q}_{\nu}\left(\mathbf{r}_{j}^{\prime}\right)
$$

This expression can be further simplified by approximating the hardness kernel as $\eta_{\mu \nu}\left(\mathbf{r}_{i}, \mathbf{r}_{j}^{\prime}\right) \approx \eta_{\mu \nu}\left(\mathbf{R}_{i}, \mathbf{R}_{j}^{\prime}\right) \equiv$ $\eta_{\mu v}(i, j)$ to obtain

$$
\mu_{\alpha}(i)=\mu_{\alpha}^{0}(i)+\sum_{v} \sum_{j} \eta_{\alpha v}(i, j) q_{v}(j),
$$

with a similar expression for $\mu_{\beta}(i)$ given by

$$
\mu_{\beta}(i)=\mu_{\beta}^{0}(i)+\sum_{v} \sum_{j} \eta_{\beta v}(i, j) q_{v}(j),
$$

where $q_{v}(j)=\int \mathrm{d} \mathbf{r}_{j} \Phi_{v}\left(\mathbf{r}_{j}\right)$ denotes the $j$ th atomic site charge. The energy change as given by (25) can be reexpressed in terms of these charges as

$$
\begin{aligned}
\Delta E= & \sum_{v} \sum_{i} \mu_{v}^{0}(i) q_{v}(i)+\frac{1}{4} \sum_{\mu} \sum_{v} \sum_{i} \sum_{j} \eta_{\mu v}(i, j) \\
& q_{\mu}(i) q_{v}(j) .
\end{aligned}
$$

The lattice model of the set of interacting atoms thus correspond to a set of point charges (zeroeth moment of the density) located at the $M$ lattice sites. An equalization of the effective chemical potential corresponding to each spin as given by (29) and (30) for all the $M$ atomic sites leads to $M-1$ linear equations in the charge variables for each spin which along with the charge conservation condition, $\sum_{j} q_{v}(j)=0$, yield the individual site charges. It may be noted that while the conventional chemical potential equalization schemes which usually consider only the net charges on atomic sites predict only zero charge and interaction among identical atoms, the present scheme which considers spin dependent charge can predict the interaction energy even for similar atoms if the atoms concerned are open shell ones. This scheme can be further generalized by considering the bond sites (say $P$ in number) between the bonded atoms in addition to the atomic sites. While the form of the final equations remains the same, the number of sites becomes $M+P$ resulting into $M+P-1$ linear equations for obtaining the site and bond charges for each spin.

The energy expression of (31) is reminiscent of the binding energy of molecules as obtained by semiempirical quantum chemistry and calculations based on this procedure lead (Ghosh 1994; Ghanty and Ghosh 1991, 1992, 1994a,b) to binding energies and partial atomic charges of simple molecular systems in good agreement with more detailed $a b$ initio calculations. The present formalism also resembles the Miedema (1973) model (Pettifor 1987) for predicting the heat of formation of simple alloy systems.

The input parameters in the present approach are the chemical potentials of the atomic (and bond) sites and also the diagonal and off-diagonal elements of the hardness matrix, $\eta_{u v}(i, j)$, corresponding to different spins and sites. The atomic chemical potentials, $\mu_{\alpha}^{0}(i)$ and $\mu_{\beta}^{0}(i)$ and the corresponding hardness parameters, $\eta_{\alpha \alpha}(i, i)$, $\eta_{\boldsymbol{\beta} \beta}(i, i)$ and $\eta_{\alpha \beta}(i, i)$ of the $i$ th atom can be obtained (Ghanty and Ghosh 1994a) from spin polarized DFT calculations. If bond sites are used, the corresponding chemical potential and hardness parameters can be approximated (Ghanty and Ghosh 1991, 1992, 1994c, 1996) by suitable averaging of the corresponding values for the 
bonded atoms. The off-diagonal $(i \neq j)$ elements of the hardness kernel, $\eta_{u v}(i, j)$, can be modeled by using the atom-in-molecule hardness matrix concept of Nalewajski et al (1996) generalized to spin-polarized situation following an electrostatic analogy (Wadehra and Ghosh 2002). The Coulomb potential approximation, $\eta_{\mu v}(i, j)=1 / \varepsilon R_{i j}$, with $\varepsilon$ as a dielectric constant, is suitable for a nonbonded pair of sites, while for bonded sites, a better modeling along the lines of semiempirical quantum chemistry as given by

$$
\eta_{\mu v}(i, j)=1 /\left(R_{i j}+a_{i j}^{\mu v}\right) \text { with } a_{i j}^{\mu v}=2 /\left(\eta_{\mu \mu}(i)+\eta_{v}(j)\right),
$$

is found to be suitable. The simplified DFT framework presented here provides a simple scheme for predicting the interatomic interaction potential and hence the forces which can be used in computer simulation as well as in mesoscopic DFT to be discussed in the next $\S$.

\section{Mesoscopic DFT: Application to soft condensed matter}

The DFT in the mesoscopic regime involves atomistic length scale and uses the single-particle number density as the basic variable. An important application that is considered here is in the area of soft condensed matter where the collective interaction and correlation effects are known to play an important role and thus the DFT formalism is a highly effective tool for the study of these many-body systems. The building blocks in this intermediate mesoscopic length scale are the atomistic particles and thus a typical system that is considered here is a twocomponent fluid mixture, with specified interparticle interaction $\left(u_{\mu v}\left(\mathbf{r}_{12}\right)\right.$ with $\mu, v=\alpha, \beta$ denoting the two components) which can be obtained either through a microscopic DFT calculation or by using simple model potentials such as hard sphere or Lennard-Jones for neutral species, charged hard sphere for ionic fluids, dipolar hard sphere for polar fluids, DLVO screened Coulomb interaction for colloids etc. In particular, our interest is in the equilibrium arrangement of the fluid particles at interfaces due to confinement of the fluid mixture, for example, in a planar slit, spherical cavity or cylindrical pore. The fluid components are subjected to the external potentials, $v_{\alpha}(\mathbf{r}), v_{\beta}(\mathbf{r})$, which lead to the inhomogeneous density distributions, $\rho_{\alpha}(\mathbf{r}), \rho_{\beta}(\mathbf{r})$, and are considered to be in equilibrium with the corresponding bulk phase of component densities, $\rho_{\alpha}^{0}, \rho_{\beta}^{0}$ and chemical potentials, $\mu_{\alpha}, \mu_{\beta}$.

The grand potential $\Omega\left[\rho_{\alpha}, \rho_{\beta}\right]$ for this system as obtained by a two-component generalization of (6) and (14), is given by

$$
\begin{aligned}
\Omega\left[\rho_{\alpha}, \rho_{\beta}\right]= & F_{\text {ex }}\left[\rho_{\alpha}, \rho_{\beta}\right] \\
& +\frac{1}{\beta_{0}} \sum_{v} \int \mathrm{d} \mathbf{r} \rho_{v}(\mathbf{r})\left\{\ln \left[\boldsymbol{\rho}_{v}(\mathbf{r}) \Lambda_{v}^{3}\right]-1\right\}
\end{aligned}
$$

$$
+\sum_{v} \int \mathrm{d} \mathbf{r}\left[v_{v}(\mathbf{r})-\boldsymbol{\mu}_{v}\right] \boldsymbol{\rho}_{v}(\mathbf{r})
$$

which on minimization followed by an equalization of the chemical potentials of the individual components with those of the bulk phases, leads to the Euler-Lagrange equations given by

$$
\begin{aligned}
\boldsymbol{\rho}_{\boldsymbol{v}}(\mathbf{r}) & =\boldsymbol{\rho}^{0} \exp \left\{-\boldsymbol{\beta}_{0} v_{v}(\mathbf{r})+c_{v}^{(1)}\left(\mathbf{r} ;\left[\boldsymbol{\rho}_{\alpha}, \boldsymbol{\rho}_{\beta}\right]\right)\right. \\
& \left.-\tilde{c}_{v}^{(1)}\left(\boldsymbol{\rho}_{\alpha}^{0}, \boldsymbol{\rho}_{\beta}^{0}\right)\right\}, \mathbf{v}=\boldsymbol{\alpha}, \boldsymbol{\beta},
\end{aligned}
$$

where the symbol $\tilde{c}$ corresponds to the bulk phase. The density equation (33) is of the Maxwellian form corresponding to an ideal gas subjected to an effective oneparticle potential contributed by the first order DCF $c_{v}^{(1)}\left(\mathbf{r} ;\left[\rho_{\alpha}, \rho_{\beta}\right]\right)$ defined as the functional derivative

$$
c_{v}^{(1)}\left(\mathbf{r} ;\left[\rho_{\alpha}, \rho_{\beta}\right]\right)=-\beta_{0} \frac{\delta F_{\mathrm{ex}}\left[\rho_{\alpha}, \rho_{\beta}\right]}{\delta \rho_{v}(\mathbf{r})},
$$

where the excess free energy, $F_{\text {ex }}\left[\rho_{\alpha}, \rho_{\beta}\right]$ contributed by interparticle correlation also defines the second order DCF $c_{v v^{\prime}}^{(2)}\left(\mathbf{r}_{1}, \mathbf{r}_{2}\right)$ through a two-component generalization of (17) as

$$
c_{v v^{\prime}}^{(2)}\left(\mathbf{r}_{1}, \mathbf{r}_{2} ;\left[\rho_{\alpha}, \rho_{\beta}\right]\right)=-\beta_{0} \frac{\delta^{2} F_{e x}\left[\rho_{\alpha}, \rho_{\beta}\right]}{\delta \rho_{v}\left(\mathbf{r}_{1}\right) \delta \rho_{v^{\prime}}\left(\mathbf{r}_{2}\right)} .
$$

In absence of knowledge of exact form of the functional $c_{v}^{(1)}\left(\mathbf{r} ;\left[\rho_{\alpha}, \rho_{\beta}\right]\right)$ for an inhomogeneous density distribution, we consider its functional Taylor expansion in powers of the density inhomogeneity, $\Delta \rho_{v}(\mathbf{r})\left[=\rho_{v}(\mathbf{r})-\right.$ $\left.\rho_{v}^{0}\right]$, as

$$
c_{v}^{(1)}\left(\mathbf{r} ;\left[\boldsymbol{\rho}_{\alpha}, \boldsymbol{\rho}_{\beta}\right]\right)=\tilde{c}_{v}^{(1)}\left(\left[\boldsymbol{\rho}_{\alpha}^{0}, \boldsymbol{\rho}_{\beta}^{0}\right]\right)+\gamma_{v}(\mathbf{r})+\Delta c_{v}(\mathbf{r}),
$$

with $\mathcal{X}_{(}(\mathbf{r})$ representing the second order contribution given by

$$
\gamma_{v}(\mathbf{r})=\sum_{v^{\prime}} \int \mathrm{d} \mathbf{r}_{2} \tilde{c}_{v v^{\prime}}^{(2)}\left(\mathbf{r}, \mathbf{r}_{2} ;\left[\rho_{\alpha}^{0}, \boldsymbol{\rho}_{\beta}^{0}\right]\right) \Delta \boldsymbol{\rho}_{v^{\prime}}\left(\mathbf{r}_{2}\right),
$$

and $\Delta c_{v}(\mathbf{r})$ representing all higher order contributions. With this prescription, the density equation (33) can be reexpressed as

$$
\rho_{v}(\mathbf{r})=\rho_{v}^{0} \exp \left[-\beta_{0} v_{v}(\mathbf{r})+\gamma_{v}(\mathbf{r})+\Delta c_{v}(\mathbf{r})\right], v=\alpha, \beta .
$$

Since the second order DCF is often known for some model pair potentials, the corresponding contribution, $\mathcal{X}_{(}(\mathbf{r})$, can easily be evaluated, but in absence of knowledge of the higher order DCFs, appropriate prescriptions are to be proposed for the quantity, $\Delta c_{v}(\mathbf{r})$. One of the simplest schemes is to approximate it as a third order contribution for which a knowledge of the third order DCF is needed. In absence of an exact form, this quantity 
has recently been approximated (Choudhury and Ghosh 1999) in terms of two-body terms in the spirit of Kirkwood's superposition approach, and also as a two-component generalization of the one-component result of Rickayzen and Augousti (1984). The parameters introduced in the approximation are evaluated by demanding the approximate density functional to yield the correct bulk partial pressures in the homogeneous limit. An alternative route has also been proposed (Choudhury and Ghosh 2001a) for evaluating $\Delta c_{v}(\mathbf{r})$ using the bridge function concept of integral equation theory of homogeneous fluid mixtures. Besides these perturbative approaches, one also has nonperturbative WDA based methods as generalization of (19) or (20) to mixtures, i.e. by using suitable effective weighted densities to evaluate the excess free energy or the first order DCF as an approximation to contributions from all orders. There are also partially nonperturbative approaches (Choudhury and Ghosh 1996a, b), where the short range contribution is evaluated using WDA, while the long range contribution is obtained through functional perturbation.

There have been many applications of these approaches dealing with the structure of fluids at interfaces and in cavities of planar, spherical or cylindrical shapes. The numerical calculations become simpler in all these cases since the density variation is along only one coordinate, leading to only one-dimensional integrations. As examples, the structure of electrode-electrolyte interface for the restricted primitive model as well as the hard sphere

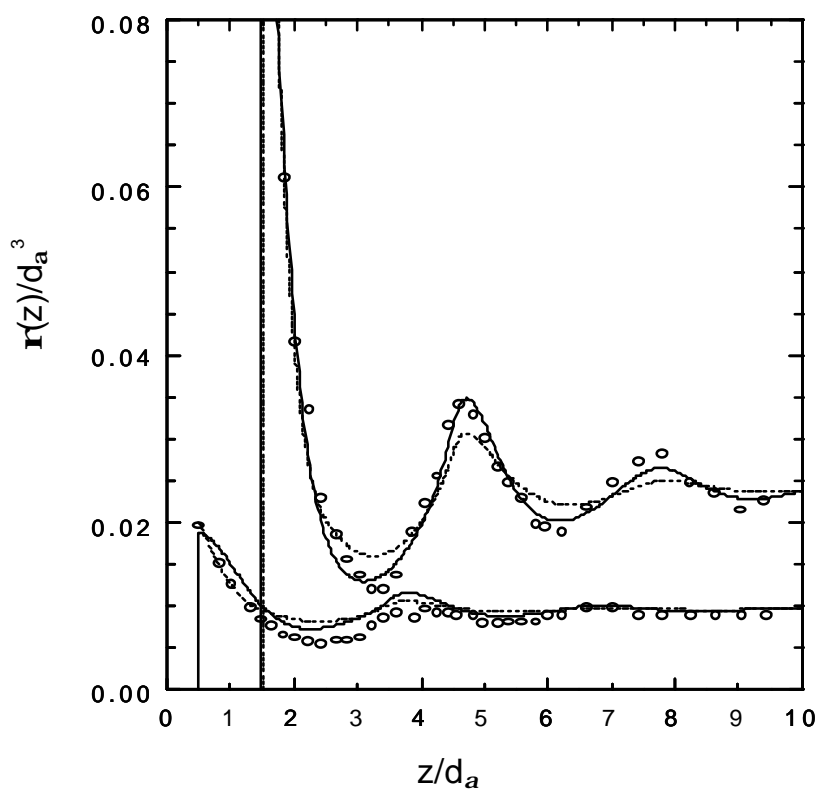

Figure 1. Plot of density profiles for a hard sphere mixture near a hard wall with packing fraction $=0.3435$, diameter ratio $=1 / 3$ and mole fraction $=0.7144$ (Bottom curves: small diameter component $(\alpha)$; upper curves: larger diameter component $(\beta)$. Solid line: Scheme of Choudhury and Ghosh (1999); dashed line: WDA of Denton and Ashcroft (1989); circles: simulation). solvent model has been predicted (Patra and Ghosh 1993a,b, 1994a,b) in good agreement with the simulation results. The long range Coulomb contribution is evaluated here through an electrostatic potential similar to that used in many-electron quantum systems, while it is only the short range correlation for which the WDA or functional perturbative method is used. The layered structure of the inhomogeneous density distribution at the interfaces (for typical plots, see figures 1 and 2) has been known to give rise to the so called solvation or structural force which is experimentally measurable. An interaction between the overlapping electric double layers is manifested into the interaction between colloidal particles in a suspension. DFT has been employed (Patra and Ghosh 1994c) to predict the solvation force for neutral as well as ionic model liquids in good agreement with simulation results as well as to reproduce (Patra and Ghosh 1994d) the experimental interaction energies between two mica walls confining a real liquid using only the hard sphere diameter as parameter. DFT has also been successfully applied to the study of sedimentation phenomena (Choudhury and Ghosh 2002) as well as adsorption isotherm (Choudhury and Ghosh 2001b) in porous medium. The effect of surface induced density inhomogeneity and the surface forces on the dynamics in condensed phase has also been of current interest.

Besides these cases of density inhomogeneities characteristic of confined fluids as a result of the corresponding external potentials, the spontaneous density

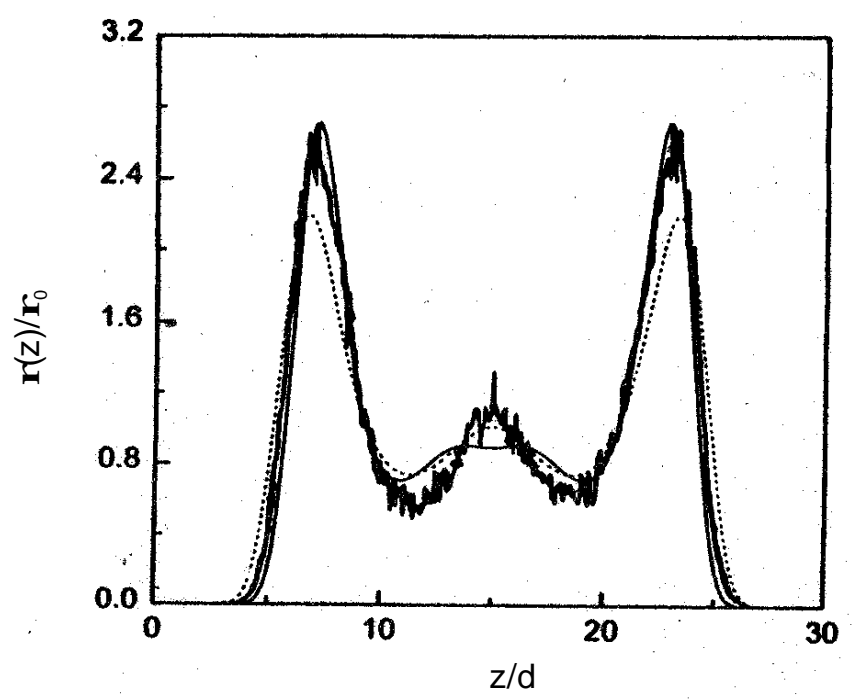

Figure 2. Plot of density profile for a model colloidal suspension in a planar slit for wall separation of $30 \mathrm{~d}$, packing fraction $=0 \cdot 00042$, screening length $=0 \cdot 15$, wall-particle coupling parameter $=190, \quad$ particle-particle coupling parameter $=580$ (Thin solid line: perturbation theory results from Choudhury and Ghosh (1998a); dashed line: hypernetted chain approximation; thick solid line: simulation). 
inhomogeneity as arising, for example, in phase transitions, through stabilization of thermal density fluctuations at suitable temperature or density parameters, has also been successfully dealt with by DFT. The original pioneering work of Ramakrishnan and Yussouff (1979) which laid the foundation of the DFT of freezing has now grown into a vast field of research. The basic theme of this DFT based approach is to consider the free energy or grand potential as density functionals and locate the physical parameters for which the ordered phase with an inhomogeneous periodic density distribution is energetically more stabilized in comparison to the fluid phase of uniform density. The Ramakrishnan and Yussouff (1979) theory has now been extensively applied to phase transition to ordered phase for a large class of soft condensed matter systems. The ordering of colloidal suspensions as predicted by DFT (Sengupta and Sood 1991; Choudhury and Ghosh 1995a) has been shown to agree quite well with experimental results. The structure of a crystal-melt interface has also been predicted (Choudhury and Ghosh 1998b) by a DFT approach by proposing a new layerwise modified WDA along with a simple modeling of the interfacial density to be periodic in the parallel plane, but decaying oscillatory in the perpendicular direction (see figure 3 for a plot of the planar-averaged density profiles for different interfacial layers). The surface energy predicted by this approach as a function of the number of interfacial layers (shown in figure 4) is found to be in good agreement with the simulation results. The DFT of freezing has also been extended to quantum systems and used, for example, for the study (Choudhury and Ghosh 1995b) of Wigner crystallization. Application of DFT to various other phase transitions, nucleation and a large class of equilibrium phenomena has also been quite successful.

\section{Density functional theory of time-dependent systems}

Although as already discussed, DFT has been immensely versatile to deal with a wide class of time-independent problems, its applicability has been further enhanced through a generalization to cover dynamical phenomena in both quantum and classical domains. Besides density, $\rho(\mathbf{r}, \mathbf{t})$ being time-dependent (TD), the additional basic variable that plays an important role in TD DFT is the single-particle TD current density, $\mathbf{j}(\mathbf{r}, \mathbf{t})$. For quantum systems, the TD DFT involves (Deb and Ghosh 1982; Runge and Gross 1984; Ghosh and Dhara 1988) the TD one-particle Kohn-Sham like equations

$$
\left[-\frac{\hbar^{2}}{2 m} \nabla^{2}+v_{\text {eff }}(\mathbf{r}, \mathbf{t})\right] \boldsymbol{\Psi}_{k}(\mathbf{r}, t)=i \hbar \frac{\partial \psi_{k}(\mathbf{r}, \mathbf{t})}{\partial t},
$$

corresponding to a system of noninteracting particles moving in a TD effective potential, $v_{\text {eff }}(\mathbf{r}, t)$, determined by the density (and also often the current density) obtained from the orbitals. There is also an equivalent quantum hydrodynamic formulation (Deb and Ghosh 1987) which consists of the continuity equation and an Euler type equation corresponding to the time evolution of the density and current density given respectively by

$$
\begin{aligned}
& \frac{\partial \rho(\mathbf{r}, \mathbf{t})}{\partial t}=\nabla \cdot \mathbf{j}(\mathbf{r}, \mathbf{t}), \\
& \frac{\partial \mathbf{j}(\mathbf{r}, \mathbf{t})}{\partial t}=-\rho(\mathbf{r}, \mathbf{t}) \nabla\left[v(\mathbf{r}, \mathbf{t})+\frac{\delta F}{\delta \boldsymbol{\rho}}\right]-\nabla \cdot\left(\frac{\dddot{\mathbf{j} j}}{\rho}\right) .
\end{aligned}
$$

These equations are reminiscent of the conventional hydrodynamic equations except that the classical force has

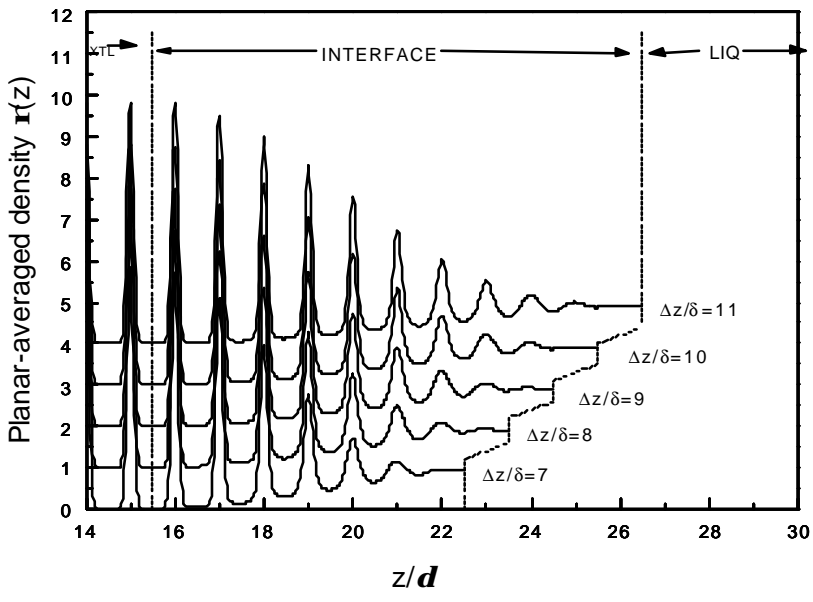

Figure 3. Plot of planar-averaged interfacial density profile for the (111) $f c c$-liquid interface of hard sphere fluid for several values of the interfacial width (Choudhury and Ghosh 1999).

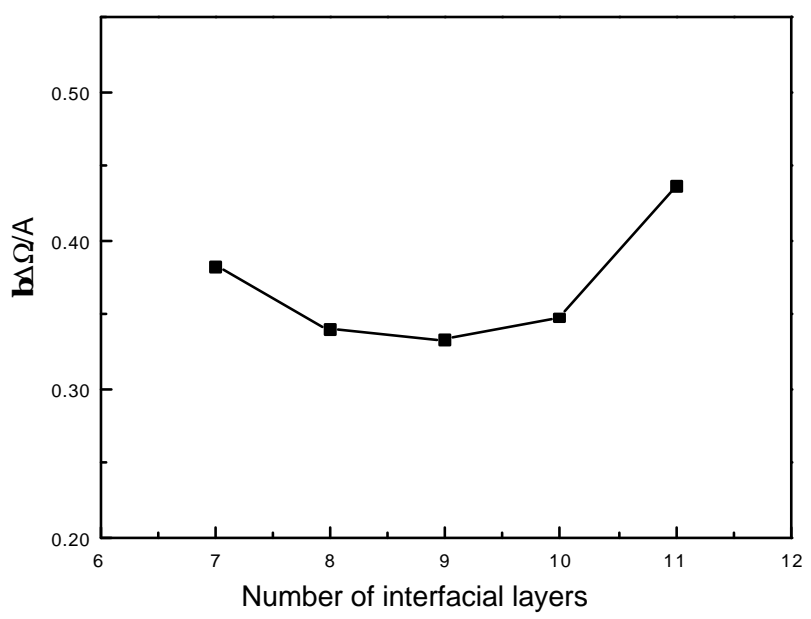

Figure 4. Plot of calculated surface free energy as a function of number of interfacial layers for (111) $f c c$-liquid interface of hard sphere fluid (Choudhury and Ghosh 1999). 
been augmented here by an additional force of quantum origin.

The TD DFT for classical systems also consist of the same continuity equation (40), but the equation for the current density evolution is given by

$$
\begin{aligned}
\frac{\partial \mathbf{j}(\mathbf{r}, \mathbf{t})}{\partial t} & =-\boldsymbol{\rho}(\mathbf{r} ; \mathbf{t}) \nabla\left[v(\mathbf{r}, \mathbf{t})+\frac{\delta F}{\delta \boldsymbol{\rho}}\right] \\
& -\int_{0}^{t} \mathrm{~d} \tau \int \mathrm{d} \mathbf{r}^{\prime} \boldsymbol{\Gamma}\left(\mathbf{r}, \mathbf{r}^{\prime} ; \mathbf{t}-\tau\right) \frac{\delta F}{\left.\delta \mathbf{j}^{\prime}, \tau\right)},
\end{aligned}
$$

where $\Gamma\left(\mathbf{r}, \mathbf{r}^{\prime} ; t\right)$ represents the dissipation kernel.

Thus, it is clear that the TD DFT for quantum and classical systems consist of closely analogous theoretical frameworks, which are suitable for bridging the microscopic and mesoscopic length scales. Extensions to spinpolarized situations for quantum TD DFT and multicomponent fluid mixtures for classical TD DFT have found wide applications to TD phenomena. A unification and interconnection of classical TD DFT with mode coupling theories (Ali et al 2001; Samanta et al 2001) of diffusion in fluid mixtures have also been demonstrated recently.

\section{Conclusions}

The picture of quantum and classical DFT encompassing the microscopic, mesoscopic as well as macroscopic length scales, has been portrayed here emphasizing the unified nature of the framework and its applicability in diverse interdisciplinary areas of materials modeling research. This is, however, only a brief and illustrative review and many important developments might not have been covered at all. DFT is a fast growing field of research and further theoretical developments along with the advancement of computational techniques are likely to play an important role in the DFT based research on multiscale materials modeling.

\section{Acknowledgements}

I am grateful to Dr G P Das for discussions and valuable suggestions. It is a pleasure to thank Drs Alok Samanta, Tapan K Ghanty, Chandra N Patra, Niharendu Choudhury, Asish K Dhara, Musharaf Ali and Amita Wadehra for their collaboration and many helpful discussions. I also thank Dr J P Mittal and Dr T Mukherjee for their interest and encouragement.

\section{References}

Ali M, Samanta A and Ghosh S K 2001 J. Chem. Phys. 114 10419

Becke A D 1993 J. Chem. Phys. 985648
Berkowitz M, Ghosh S K and Parr R G 1985 J. Am. Chem. Soc. 1076811

Car R and Parrinello M 1985 Phys. Rev. Lett. 552471

Choudhury N and Ghosh S K 1995a Phys. Rev. E51 4503

Choudhury N and Ghosh S K 1995b Phys. Rev. B51 2588

Choudhury N and Ghosh S K 1996a J. Chem. Phys. 104 9563

Choudhury N and Ghosh S K 1996b Phys. Rev. E53 3847

Choudhury N and Ghosh S K 1998a J. Chem. Phys. 1087493

Choudhury N and Ghosh S K 1998b Phys. Rev. E57 1939

Choudhury N and Ghosh S K 1999 J. Chem. Phys. 1108628

Choudhury N and Ghosh S K 2001a J. Chem. Phys. 1148530

Choudhury N and Ghosh S K 2001b Phys. Rev. E64 21206

Choudhury N and Ghosh S K 2002 J. Chem. Phys. 116384

Deb B M and Ghosh S K 1982 J. Chem. Phys. 77342

Deb B M and Ghosh S K 1987 in Single particle density in physics and chemistry (eds) N H March and B M Deb (New York: Academic)

Debye P and Huckel E 1923 Z. Phys. 24 185, 305

deGennes P G 1992 Rev. Mod. Phys. 64645

deGennes P G 1999 Rev. Mod. Phys. 71 S374

Denton A R and Ashcroft N W 1989 Phys. Rev. A39 426

Evans R 1992 in Fundamentals of inhomogeneous fluids (ed.) D Henderson (New York: Marcel Dekker) Ch. 3

Fermi E 1928 Z. Phys. 4873

Ghanty T K and Ghosh S K 1991 J. Phys. Chem. 956512

Ghanty T K and Ghosh S K 1992 Inorg. Chem. 311951

Ghanty T K and Ghosh S K 1994a J. Am. Chem. Soc. 116 8801

Ghanty T K and Ghosh S K 1994b J. Phys. Chem. 981840

Ghanty T K and Ghosh S K 1994c J. Phys. Chem. 989197

Ghanty T K and Ghosh S K 1996 J. Phys. Chem. 10017429

Ghosh S K 1990 Chem. Phys. Lett. 17277

Ghosh S K 1994 Int. J. Quant. Chem. 49239

Ghosh S K 2002 in Reviews in modern quantum chemistry (ed.) K D Sen (Singapore: World Scientific)

Ghosh S K and Deb B M 1982 Phys. Rep. 921

Ghosh S K and Dhara A K 1988 Phys. Rev. A38 1149

Henderson D (ed.) 1992 Fundamentals of inhomogeneous fluids (New York: Marcel Dekker)

Hohenberg P and Kohn W 1964 Phys. Rev. 136 B864

Kohn W and Sham L J 1965 Phys. Rev. 140 A1133

Lee C, Yang W and Parr R G 1988 Phys. Rev. B37 785

March N H and Deb B M (eds) 1987 Single particle density in physics and chemistry (New York: Academic)

Mermin N D 1965 Phys. Rev. 137 A1441

Miedema A R 1973 J. Less Common Met. 32117

Nalewajski R F, Korchowiec J and Michalak A 1996 Top. Curr. Chem. 18325

Parr R G and Yang W 1989 Density functional theory of atoms and molecules (New York: Oxford Univ. Press)

Parr R G and Yang W 1995 Annu. Rev. Phys. Chem. 46701

Parr R G, Donnelly R A, Levy M and Palke W E 1978 J. Chem. Phys. 683801

Patra C N and Ghosh S K 1993a Phys. Rev. E47 4088

Patra C N and Ghosh S K 1993b Phys. Rev. E48 1154

Patra C N and Ghosh S K 1994a J. Chem. Phys. 1005219

Patra C N and Ghosh S K 1994b J. Chem. Phys. 1014143

Patra C N and Ghosh S K 1994c Phys. Rev. E49 2826

Patra C N and Ghosh S K 1994d Phys. Rev. E50 5123

Pettifor D G 1987 Solid State Phys. 4043 
Ramakrishnan T V and Yussouff M 1979 Phys. Rev. B19 2775

Rickayzen G and Augousti A 1984 Mol. Phys. 521355

Runge E and Gross E K U 1984 Phys. Rev. Lett. 52997

Samanta A, Ali M and Ghosh S K 2001 Phys. Rev. Lett. 87 245901
Sengupta S and Sood A K 1991 Phys. Rev. A44 1233

Tarazona P 1984 Mol. Phys. 5281

Tarazona P 1985 Phys. Rev. A31 2672

Thomas L H 1927 Proc. Camb. Philos. Soc. 23542

Wadehra A and Ghosh S K 2002 (to be published)

York D M and Yang W 1996 J. Chem. Phys. 104159 